\title{
Development of an Automated and Dedicated Measuring System for Straightness Evaluation
}

Escola de Engenharia de São Carlos

Av. Trabalhador Sancarlense, 400

13566- 590 São Carlos, SP. Brasil

\section{Benedito Di Giacomo} bgiacomo@sc.usp.br

\author{
Roberto H. Tsunaki \\ rtsunaki@sc.usp.br
}

This work presents an automated and dedicated system aiming at the measurement of straightness errors of mechanical components, using an industrial robot. A multi-probe error separation technique was used to make measurements independent from the coordinate system of the robot. A mathematical model that takes into account the readings from three sensors was specifically designed for the proposed measurements and produces inspection results by means of the solution of a system of linear equations, in only one operation. Also in this work, a new approach was developed to minimize the influence of the zero-adjustment errors of the sensors, which represent the major source of errors in the separation process. Experimental tests applied to the measurement of straightness errors of mechanical components were accomplished, which demonstrated the effectiveness of the employed methodology.

Keywords: Error separation, straightness error, multi-probe method, measurement automation

\section{Introduction}

The ability to accomplish precise measurements is vitally important to the mechanical industry, where the knowledge of dimensions is essential to guarantee conformity of parts and to control the production processes. The application of automatic systems in tasks formerly made only by the human operator has hugely expanded in the last two decades, to some extent due to the growing market of $\mathrm{CNC}$ machines. On the other hand, great part of the measuring processes that are effectively done on manufacture lines until today rely on the utilization of manual instruments and conventional techniques. Therefore, the application of automated manufacturing processes requires the project and construction of also automated measuring systems, which could be integrated to the manufacturing processes.

As a result of the development of better machine tools and enhanced processes, higher quality products could be manufactured. Such products, which are often made under tighter tolerances, also require the improvement of measuring instruments making them capable of taking satisfactory measurements within the dimensional limits defined by design. Finally, the existence of accurate measuring instruments allows the construction of new and enhanced machines, initiating a favourable cycle of technological development of machines, instruments and assorted processes.

Automatic measuring instruments are especially useful for complete measurement of large lots of parts aiming at selective assembly. When compared to conventional techniques, automatic measurement can be fast and objectively accomplished. For workpieces that were machined within tight tolerances, automatic measurement offers the advantage of eliminating the influence of the human operator upon measurement. In the case of dimensional evaluation of relatively simple workpieces, such as guideways and plane or cylindrical surfaces, besides automation, it might be desirable to use a dedicated instrument with ability to evaluate a single mechanical feature much more effectively when compared to universal instruments.

In addition to the utilization of automatic machine tools, a high degree of industrial automation has been achieved by using robots in production and assembly lines. Nowadays, it is very common to find robots performing repetitive low-accuracy tasks, as painting, welding and loading/unloading. The relatively low acquisition and

Paper accepted October, 2007. Technical Editor: Anselmo E. Diniz. maintenance costs of robots, easy programming and fast execution of the programmed tasks make robots an attractive investment.

The application of industrial robots as measuring instruments, however, is not trivial. The manipulator low positioning accuracy is a critical restriction. Currently available accuracy levels at the industrial robot technology, in general, are not enough to provide reliable results in case of measurements referring back to the robot coordinate system as a reference. Factors such as tolerances of parts that compose the robot structure, elasticity at joints, resolution of resolvers, limitations of the control system, among others, produce a unique behaviour in each robot, which even limits the application of error compensation techniques.

About the possibility of adapting a piece of measuring equipment to the wrist of a robot set in order to take measurements, Greenway (2000) comments: "Conceptually, a measurement device is mounted to the end-effector of a robot that moves this device into position to take a measurement. All measurements are related back to robot position; the measurement accuracy will only be as accurate as the robot positioning". The relation between measurements and robot position disregards the device as a base to a measuring system.

In order to make measurements independent from the reference system of the robot, it is necessary to employ some error separation technique that allows decoupling between the errors of the artefact and those originated from the robotic measuring system.

The application of error separation techniques is especially desired when errors of the measuring system are not negligible if compared to workpiece errors. In these cases, it is a mistake to consider the instrument as reference.

The reversal technique, whose origin dates back to the decade of 1940 , is perhaps the most recognizable error separation technique and was developed to evaluate machine tools produced at that time (Bryan and Carter, 1989). The work by Evans, Hocken and Estler (1996) presents a very comprehensive review of various reversal techniques, which are applicable to a wide range of common situations in the mechanical industry.

Compared to reversals, multi-probe error separation methods present the advantage of not having the need of artefact manipulation. Multi-probe methods require the acquisition of redundant data on the part and besides, depending upon the type of the measured error, a specific sensorial arrangement is needed. Whitehouse (1976) describes the theory behind both multiorientation and multi-probe methods.

Several multi-probe methods were proposed to accomplish straightness error measurement and the most prominent are the twoprobe method (Tanaka et al. 1981; Gao and Kiyono, 1996) and the 
three-probe method (Tanaka and Sato, 1986; Gao and Kiyono, 1997). An issue arises as the three-probe method is extremely sensitive to the presence of zero-adjustment errors of the probes. Gao et al. (2002) have shown that the difference between the zeroreadings of the probes introduces a parabolic error term in the profile result, which consists of the largest influence source in determining straightness error by three points measurement.

In this work, an automated and dedicated multi-probe measuring system is presented. The system is basically composed of an industrial robot, specifically arranged displacement sensors and a multi-probe error separation mathematical model. A self-calibration procedure was developed to minimize the influence of probes zeroadjustment errors. Experimental tests were performed and proved the efficiency of the proposed technique.

\section{Nomenclature}

G3P $=$ Generalized Three Points method

$\mathrm{k}=$ zero-adjustment error, $\mu \mathrm{m}$

$\mathrm{L}=$ distance between sensors, $\mathrm{mm}$

$\mathrm{M}=$ total number of measurement runs

S3P $=$ Sequential Three Points method

$\mathrm{S}_{\mathrm{A}}, \mathrm{S}_{\mathrm{B}}, \mathrm{S}_{\mathrm{C}}=$ readings of sensors $\mathrm{A}, \mathrm{B}$ and $\mathrm{C}, \mu \mathrm{m}$

$R_{P}=$ straightness error of profile, $\mu \mathrm{m}$

$\mathrm{R}_{\mathrm{R}}=$ robot translational error motion, $\mu \mathrm{m}$

$\mathrm{x}, \mathrm{y}, \mathrm{z}=$ coordinate system

\section{Greek Symbols}

$\delta=$ displacement due to pitch of scanning device, $\mu m$

$\theta=$ pitch error of scanning device, $\mathrm{rad}$

\section{Subscripts}

$A, B, C$ sensor index

$i \quad$ relative to the number of measuring points

$P$ relative to artefact straightness error

$R$ relative to robot translational error motion

\section{Superscripts}

$D$ relative to the contribution of the displacement due to pitch

$E$ relative to the contribution of the zero-adjustment error

$h$ relative to the number of reference lines for the straightness error of the artefact considered in the error model

$j$ relative to the number of measurement runs

$k$ relative to the number of robot translational error motion values considered in the error model

$P$ relative to the contribution of the artefact straightness error

$R$ relative to the contribution of the robot translational error motion

$s \quad$ relative to the number of reference lines for the robot translational error motion considered in the error model

$t$ relative to the number of displacements due to pitch of scanning device considered in the error model

$v$ relative to the number of zero-adjustment error values considered in the error model

\section{The Utilization of Robots as Inspection Devices}

During the selection of a measuring system, several factors must be considered, for instance, the verified geometry, the required tolerance levels, the number of parts to be tested, the degree of complexity of parts and the ability of reconfiguration to meet possible modifications in the production line. Figure 1 indicates that a measuring system can be determined taking into account the lot size and the variety of parts to be verified (Hocken, 1995).

Coordinate measuring machines (CMM) are perhaps the most flexible measuring instruments, presenting the ability of measuring
Cartesian coordinates of spatial points by means of scales and, frequently, a touch-trigger type probe, which can be replaced by laser scanning probes or CCD cameras. Current models present resolution of about $0.1 \mathrm{~mm}$. Complex geometry workpieces can be evaluated rather straightforwardly with CMMs. Considering the case of large lots of relatively simple geometry parts, dedicated and automated measuring instruments are potentially more efficient, enabling inspection of the full lot. Grant (1995) considers that total inspection is undesirable, since it would indicate that the process is prone to errors. However, Boillot and Uota (2002) state that the aim of total inspection is not to compensate for poor process, but rather to identify very defective parts. Moreover, total inspection can be used to classify and separate parts for selective assembly. In this case, a CMM is not so effective as an automated and dedicated measuring system.

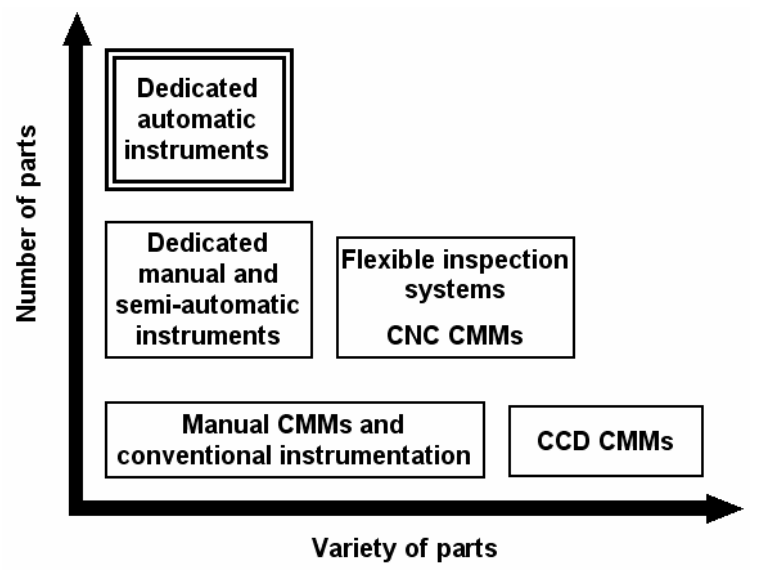

Figure 1. Selection of measuring system (Hocken in Bosch, 1995).

Additional aspects can yet be considered in the implementation of a robotic measuring system: firstly, industries aim the increase of product quality by enhancing manufacturing process control. This action is simpler when using automatic systems due to the exclusion of human effects. Another factor to be considered is the velocity at the execution of a task. As long as the robot can provide velocity, larger productivity can be attained.

About the utilization of articulated arms, Hoshizaki and Bopp (1990) consider that repeatability and accuracy of this configuration are not good enough to allow the realization of precision tasks. As a matter of fact, robot accuracy, which is rarely quoted on manufacturers booklets, can sometimes be several orders of magnitude worse than positioning repeatability (Young e Pickin, 2000).

Kato, Sone and Nomura (1991) presented a measuring system that consisted of a robot and one displacement sensor for evaluation of roundness errors on cross sections of cylindrical parts. The robot was employed to place the transducer around the workpiece. Error value was calculated by means of the three points method for circularity (Mitsui, 1982), yielding to satisfactory results.

In the field of non-contact measurement, several industries have been employing laser scanning devices on the wrist of robots in order to accomplish so said "more precise" measurements. However, measurement accuracy is limited by the accuracy of the robot itself, which is around $100 \mathrm{~mm}$. Thus, using robot position as reference must be carefully planned and only done when convenient. Some examples of this type of application, which consider low precision tasks, are described next: Lee and Park (2000) developed algorithms for inspection of free-form shape workpieces by laser scanning. Boillot e Uota (2002) used an industrial robot and a laser scanning device to verify weldments on truck frames. Picard (2002) 
developed an algorithm to evaluate and correct the positioning of vehicle parts to be assembled by robots.

It can be observed that robotic measuring systems are almost always directed to the verification of large tolerances due to the absence of an adequate reference to measurements. In order to make measurements independent from the robot accuracy, the application of some error separation technique becomes necessary, since artefact errors can be decoupled from the motion errors of the robot system.

\section{Multi-Probe Error Separation Methods}

All multi-probe error separation techniques require the manipulation of a selected degree-of-freedom of the system other than the sensitive direction of the sensor. This operation changes the phase of one component of the error (Evans, Hocken and Estler, 1996). The Two-Probe Method, which provides separation of probe motion straightness error from artefact straightness error, is the simplest multi-probe method (Tanaka et al. 1981; Gao and Kiyono, 1996). Two displacement sensors are moved along the measuring path, scanning the artefact in regular intervals that correspond to the distance between probes. The two-probe method, however, is unable to eliminate possible deleterious influences from rotational movements of the probing device.

The Three-Probe Method, described next, is capable of eliminating the influence of rotational and translational errors of the probing system, allowing detection of part straightness profile free from the deterioration caused by the measuring system. Figure 2 shows the operational principle of the three-probe method.

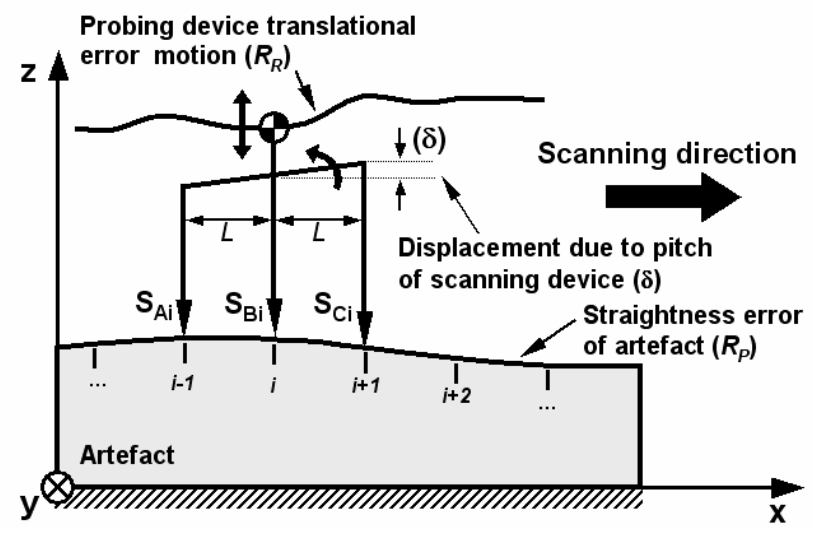

Figure 2. Three-Point Method operational principle schematic.

The readings of sensors $A, B$ and $C$, represented by $S_{A}, S_{B}$ and $S_{C}$, can be expressed as a function of system errors at position $i$ : Let $R_{P i}$ be the straightness error of the artefact to be measured, $R_{R i}$ the probing system translational error motion and $\delta_{i}$ and $-\delta_{i}$ respective displacement at probes $A$ and $C$ tips due to pitch of the probing device on the scanning direction. When the scanning step is equal to the distance between sensors $(L)$, the three-probe method is referred to as the S3P method (Sequential Three Points) (Tanaka and Sato, 1986). Otherwise, when scanning step is smaller than $L$, the threeprobe method is referred to as the G3P method (Generalized Three Points) (Gao and Kiyono, 1997). In the absence of probe calibration errors, both three-point methods allow perfect identification of artefact profile from the collected data. In the $S 3 P$ method, sensor readings can be expressed as:

$$
\left\{\begin{array}{l}
S_{A_{i}}=R_{P_{i-1}}+R_{R_{i}}-\delta_{i} \\
S_{B_{i}}=R_{P_{i}}+R_{R_{i}} \\
S_{C_{i}}=R_{P_{i+1}}+R_{R_{i}}+\delta_{i}
\end{array}\right.
$$

The main error source in the three-probe method consists of the impossibility of providing a global reference for the probes zerovalue. The presence of zero-adjustment errors originates a parabolic error term in the profile result of the measured artefact. In addition, probe position cannot be easily adjusted even with the help of a sufficiently accurate reference flat surface (Gao et al., 2002). Figure 3 illustrates the zero-adjustment errors of the three sensors in the probing device. Constants $k_{A}, k_{B}$ and $k_{C}$ correspond to the adjustment errors over the readings of sensors $A, B$ and $C$, respectively.

The proposed multi-probe technique for straightness measurement is described next. As well as the S3P method, the proposed technique requires that experimental data is collected at steps that correspond to the distance between sensors.

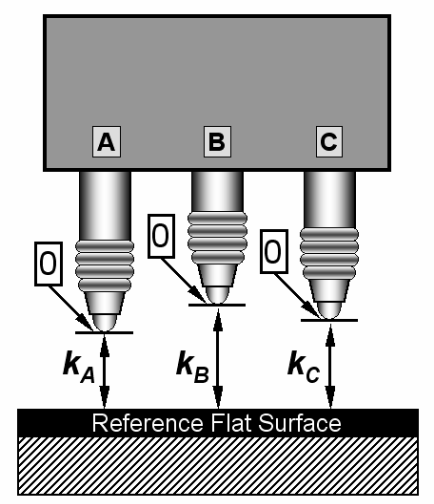

Figure 3. Zero-adjustment errors.

A mathematical model can be developed from the sensors output signals if the hypothesis that four error sources are superimposed at the output is considered: 1) errors due to workpiece straightness profile; 2) errors due to probing device translational error motion along the measuring path, 3) errors due to probing device pitch and 4) zero-adjustment errors. Hence, each sensor output can be expressed as the superposition of the four referred error sources:

$$
\begin{gathered}
\left\{\begin{array}{c}
S_{A_{i}}=S_{A_{i}}^{P}+S_{A_{i}}^{R}+S_{A_{i}}^{D}+S_{A_{i}}^{E} \\
S_{B_{i}}=S_{B_{i}}^{P}+S_{B_{i}}^{R}+S_{B_{i}}^{D}+S_{B_{i}}^{E} \\
S_{C_{i}}=S_{C_{i}}^{P}+S_{C_{i}}^{R}+S_{C_{i}}^{D}+S_{C_{i}}^{E}
\end{array}\right. \\
i=1,2, \ldots, N
\end{gathered}
$$

where:

$S_{A_{i}}, S_{B_{i}}, S_{C_{i}}$ are the $i^{\text {th }}$ readings of sensors $A, B$ and $C$, respectively;

$S_{A_{i}}^{P}, S_{B_{i}}^{P}, S_{C_{i}}^{P}$ are the workpiece straightness errors at the $i^{\text {th }}$ measuring point, detected by sensors $A, B$ and $C$, respectively; $S_{A_{i}}^{R}, S_{B_{i}}^{R}, S_{C_{i}}^{R}$ are the translational error motion of the industrial robot, which is the base of the measuring system, at the $i^{\text {th }}$ measuring point, on sensors $A, B$ and $C$, respectively;

$S_{A_{i}}^{D}, S_{B_{i}}^{D}, S_{C_{i}}^{D}$ are the contributions due probing device pitch at the $i^{\text {th }}$ measuring point, on sensors $A, B$ and $C$, respectively; $S_{A_{i}}^{E}, S_{B_{i}}^{E}, S_{C_{i}}^{E}$ are the contributions due to the zero-adjustment errors at the $i^{\text {th }}$ measuring point, on sensors $A, B$ and $C$, respectively; 
$i$ indicates a measuring point;

$N$ is the total number of measurements.

An additional hypothesis, which is implicit in Eq.(2), is that each contribution can be individually analysed, i.e., one error source does not alter the behaviour of the others, within reasonable limits that do not affect the overall desired accuracy level. As a consequence, each term of Eq.(2) is independent and can be separately developed. It must be observed that there is no restriction as to amplitude and relative proportion among all contributions.

\section{Errors Due to Workpiece Profile}

The portion of the probes output signals due to workpiece shape and misalignment at the $i^{\text {th }}$ measured value can be defined by:

$$
\left\{\begin{array}{l}
S_{A_{i}}^{P}=R_{P_{i-1}}+\left[C A_{P}+A_{P} \cdot x_{i-1}\right] \\
S_{B_{i}}^{P}=R_{P_{i}}+\left[C A_{P}+A_{P} \cdot x_{i}\right] \\
S_{C_{i}}^{P}=R_{P_{i+1}}+\left[C A_{P}+A_{P} \cdot x_{i+1}\right] \\
i=1,2, \ldots, N
\end{array}\right.
$$

where:

$x_{i}$ is the coordinate that represents the $i^{\text {th }}$ position along the workpiece profile;

$R_{P_{i}}$ is the workpiece straightness error at the $i^{\text {th }}$ measuring position;

$C A_{P}$ and $A_{P}$ are the parameters of the reference line (with relation to a reference system) of the workpiece profile.

The system shown in Eq.(3) can be simplified given the discrete character of sensor positioning on the profile and also that scanning step is constant and equal to the distance between sensors. A change of coordinates can be made so that index $i$ is used instead of position $x$, which yields:

$$
\left\{\begin{array}{c}
S_{A_{i}}^{P}=R_{P_{i-1}}+\left[C A_{P}+A_{P} \cdot(i-1)\right] \\
S_{B_{i}}^{P}=R_{P_{i}}+\left[C A_{P}+A_{P} \cdot(i)\right] \\
S_{C_{i}}^{P}=R_{P_{i+1}}+\left[C A_{P}+A_{P} \cdot(i+1)\right] \\
i=1,2, \ldots, N
\end{array}\right.
$$

A similar model can be developed to cover step lengths that are different (especially smaller) than the distance between sensors, but it will not be considered in this work.

\section{Errors Due to Robot Translational Motion along the Measuring Path}

The contribution of the robot translational error motion along the measuring path upon sensor readings can be expressed in the same way as the workpiece profile:

$$
\left\{\begin{array}{c}
S_{A_{i}}^{R}=R_{R_{i}}+\left[C A_{R}+A_{R} \cdot x_{i-1}\right] \\
S_{B_{i}}^{R}=R_{R_{i}}+\left[C A_{R}+A_{R} \cdot x_{i-1}\right] \\
S_{C_{i}}^{R}=R_{R_{i}}+\left[C A_{R}+A_{R} \cdot x_{i-1}\right] \\
i=1,2, \ldots, N
\end{array}\right.
$$

where:

$R_{R_{i}}$ is the translational error motion of the industrial robot at the $i^{\text {th }}$ measuring position;
$C A_{R}$ and $A_{R}$ are the robot translational error motion reference line parameters.

Again, the system shown in Eq.(5) can be simplified by a change of coordinates:

$$
\left\{\begin{array}{c}
S_{A_{i}}^{R}=R_{R_{i}}+\left[C A_{R}+A_{R} \cdot(i-1)\right] \\
S_{B_{i}}^{R}=R_{R_{i}}+\left[C A_{R}+A_{R} \cdot(i-1)\right] \\
S_{C_{i}}^{R}=R_{R_{i}}+\left[C A_{R}+A_{R} \cdot(i-1)\right] \\
i=1,2, \ldots, N
\end{array}\right.
$$

The translational error motion affects all three sensors on a given measuring point by the same amount, i.e., $S_{A_{i}}^{R}=S_{B_{i}}^{R}=S_{C_{i}}^{R}$.

\section{Errors Due to Probing Device Pitch along the Measuring Path}

The error produced by the scanning device pitch during the measuring procedure can be expressed as follows:

$$
\left\{\begin{array}{l}
S_{A_{i}}^{D}=-\delta_{i} \\
S_{B_{i}}^{D}=0 \\
S_{C_{i}}^{D}=+\delta_{i} \\
\quad i=1,2, \ldots, N
\end{array}\right.
$$

where $\delta_{i}$ corresponds to the probing device angular error at the $i^{\text {th }}$ measuring position, which is given by:

$$
\delta_{i}=\theta_{i} \cdot L
$$

where:

$\theta_{i}$ is the probing device pitch error in radians;

$L$ is the distance between sensors.

In this case, it is supposed that the rotation happens around the central sensor. Thus, the angular error produces an increase in $S_{A_{i}}^{D}$ and a corresponding decrease of same value in $S_{C_{i}}^{D}$, whilst $S_{B_{i}}^{D}$ remains unchanged.

\section{Zero-Adjustment Errors and the Proposed Model}

The most influent error source on the three-point method is the lack of a single reference for all sensors in the scanning device.

A calibration procedure could be carried out using a reference flat surface in order to obtain an ideal alignment among sensors, as shown in Fig. 4(a). However, minimal discrepancies between the zero values of the three sensors (with relation to an ideal flat surface) produce a large deleterious parabolic error term on the final profile result (Gao et al., 2002).

The zero-adjustment error can be expressed in several ways, depending on how the reference is chosen. It can be reduced to one adjustment component (calibration constant $k_{B}$ ) on the central sensor if sensors $A$ and $C$ zero-readings were taken as reference points, as illustrated in Fig. 4(b). 


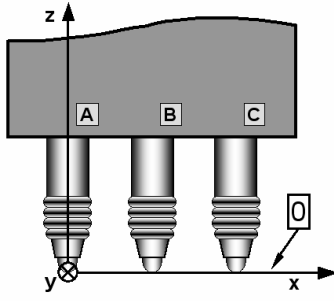

(a)

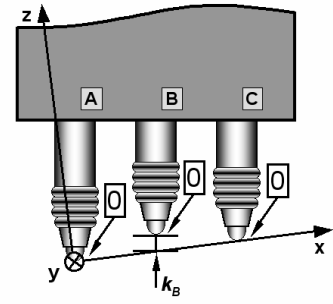

(b)
Figure 4. Ideal alignment (a) and zero-adjustment error, $\boldsymbol{k}_{B}(\mathrm{~b})$

The choice of sensors $A$ and $C$ zero-readings as reference also settles the orientation of a coordinate system. Such orientation is described by two orthogonal axes. $X$ axis is defined by the line intercepting the reference points at sensors $A$ and $C$. $Z$ axis is defined by a line that is normal to axis $X$. The system origin coincides with the first point of the workpiece straightness error, which is identically equal to zero as imposed by the model.

Therefore, the contribution due to zero-adjustment errors can be expressed as:

$$
\left\{\begin{array}{l}
S_{A_{i}}^{E}=0 \\
S_{B_{i}}^{E}=k_{B} \\
S_{C_{i}}^{E}=0
\end{array}\right.
$$

$$
i=1,2, \ldots, N
$$

where $k_{B}$ is a constant that describes the zero-adjustment error of the central sensor $B$ with relation to a reference system originated from the zero-readings of sensors $A$ and $C$. Next, the proposed coordinate system will be used to define the error model. Substituting Eqs.(4), (6), (7) and (9) in Eq. (2) yields:

$$
\begin{aligned}
& \left\{\begin{array}{l}
S_{A_{i}}=R_{P_{i-1}}+\left[C A_{P}+A_{P} \cdot(i-1)\right]+R_{R_{i}}+\left[C A_{R}+A_{R} \cdot(i-1)\right]-\delta_{i} \\
S_{B_{i}}=R_{P_{i}}+\left[C A_{P}+A_{P} \cdot i\right]+R_{R_{i}}+\left[C A_{R}+A_{R} \cdot(i-1)\right]+k_{B} \\
S_{C_{i}}=R_{P_{i+1}}+\left[C A_{P}+A_{P} \cdot(i+1)\right]+R_{R_{i}}+\left[C A_{R}+A_{R} \cdot(i-1)\right]+\delta_{i}
\end{array}\right. \\
& i=1,2, \ldots, N
\end{aligned}
$$

Considering a data set of $N$ sensor readings, the following system of equations can be derived:

$$
\begin{aligned}
& S_{A_{1}}=R_{P_{0}}+C A_{P}+R_{R_{1}}+C A_{R}-\delta_{1} \\
& S_{B_{1}}=R_{R}+C A P+A P+R_{R_{1}}+C A_{R}+k_{B} \\
& S_{C_{1}}=R_{P_{2}}+C A P+2 A P+R_{R_{1}}+C A_{R}+\delta_{1} \\
& \bar{S}_{A_{2}}=\bar{R}_{1}+\bar{C} A_{P}+\bar{A}_{P}+R_{R_{2}}+\overline{C A}_{R}+\bar{A}_{R}-\delta_{2} \\
& S_{B_{2}}=R_{P_{2}}+C A_{P}+2 A_{P}+R_{R_{2}}+C A_{R}+A_{R}+k_{B} \\
& S_{C_{2}}=R_{P_{3}}+C A_{P}+3 A_{P}+R_{R_{2}}+C A_{R}+A_{R}+\delta_{2} \\
& \left\{S_{A_{3}}=R_{P_{2}}+C \bar{A}_{P}+2 \bar{A}_{P}+R_{R_{3}}+\overline{C A}_{R}+2 \bar{A}_{R}-\delta_{3}\right. \\
& S_{B_{3}}=R_{P_{3}}+C A_{P}+3 A_{P}+R_{R_{3}}+C A_{R}+2 A_{R}+k_{B} \\
& S_{C_{3}}=R_{P_{4}}+C A P+4 A P+R_{R_{3}}+C A_{R}+2 A_{R}+\delta_{3} \\
& \left.\overline{S_{A_{N}}}=\bar{R}_{P_{N-1}}+[\bar{C} \bar{A} \bar{P}+\bar{A} \bar{P} \cdot(\bar{N}-\overline{1})]+\bar{R}_{R_{N}}+\left[\bar{C} \bar{A}_{R}+\bar{A} \bar{R} \cdot \overline{(N}-\overline{1}\right)\right]-\overline{\delta_{N}} \\
& S_{B_{N}}=R_{P_{N}}+\left[C A_{P}+A_{P} \cdot N\right]+R_{R_{N}}+\left[C A_{R}+A_{R} \cdot(N-1)\right]+k_{B} \\
& S_{C_{N}}=R_{P_{N+1}}+\left[C A_{P}+A_{P} \cdot(N+1)\right]+R_{R_{N}}+\left[C A_{R}+A_{R} \cdot(N-1)\right]+\delta_{N}
\end{aligned}
$$

The dashed lines in the system in Eq.(11) distinguish the equations written at a common measuring position on the workpiece. Eq.(11) can be rearranged gathering equations containing the same straightness error terms:

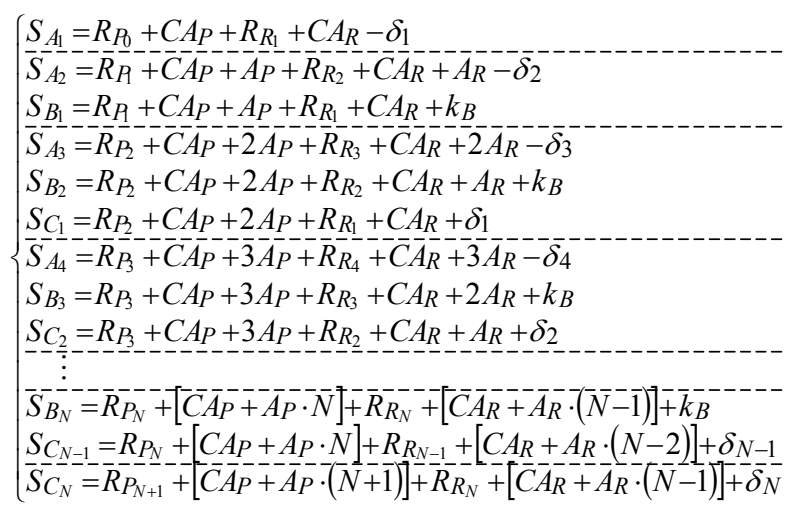

Again, the system above can be rewritten so as to gather the equations whose readings were taken by the same sensor.

$$
\begin{aligned}
& S_{A_{1}}=R_{P_{0}}+C A_{P}+R_{R_{1}}+C A_{R}-\delta_{1} \\
& S_{A_{2}}=R_{1}+C A_{P}+A_{P}+R_{R_{2}}+C A_{R}+A_{R}-\delta_{2} \\
& S_{A_{3}}=R_{P_{2}}+C A_{P}+2 A_{P}+R_{R_{3}}+C A_{R}+2 A_{R}-\delta_{3} \\
& S_{A_{4}}=R_{P_{3}}+C A_{P}+3 A_{P}+R_{R 4}+C A_{R}+3 A_{R}-\delta_{4}
\end{aligned}
$$

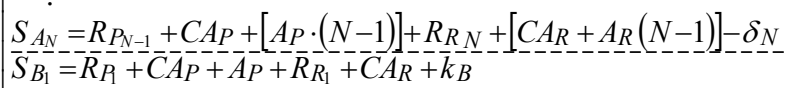

$$
\begin{aligned}
& S_{B_{2}}=R_{P_{2}}+C A_{P}+2 A_{P}+R_{R_{2}}+C A_{R}+A_{R}+k_{B} \\
& S_{B_{3}}=R_{P_{3}}+C A_{P}+3 A_{P}+R_{R_{3}}+C A_{R}+2 A_{R}+k_{B} \\
& \left\{\begin{array}{l}
S_{B_{4}} \\
:
\end{array}\right. \\
& S_{B_{N}}=R_{P_{N}}+C A_{P}+\left[A_{P} \cdot N\right]+R_{R_{N}}+\left[C A_{R}+A_{R} \cdot(N-1)\right]+k_{B} \\
& \bar{S}_{C_{1}}=\bar{R}_{P_{2}}+\bar{C} A_{P}+\overline{2} A_{P}+\bar{R}_{R_{1}}+\bar{C} \bar{A}+\bar{\delta}_{1} \\
& S_{C_{2}}=R_{P_{3}}+C A_{P}+3 A_{P}+R_{R_{2}}+C A_{R}+A_{R}+\delta_{2} \\
& S_{C_{3}}=R_{P_{4}}+C A P+4 A P+R_{R_{3}}+C A_{R}+2 A_{R}+\delta_{3} \\
& S_{C_{4}}=R_{P_{5}}+C A_{P}+5 A_{P}+R_{R 4}+C A_{R}+3 A_{R}+\delta_{4} \\
& S_{C_{N}}=R_{P_{N+1}}+C A_{P}+\left[A_{P} \cdot(N+1)\right]+R_{R N}+\left[C A_{R}+A_{R}(N-1)\right]+\delta_{N}
\end{aligned}
$$

An analysis of the system depicted in Eq.(13) reveals the number of equations and unknowns:

The number of unknowns is equal to $3 N+7$, which are detailed below:

$R_{P_{0}}$ to $R_{P_{N+1}} \rightarrow N+2$ unknowns;

$R_{R_{1}}$ to $R_{R_{N}} \rightarrow N$ unknowns;

$C A_{P}, A_{P}, C A_{R}, A_{R}$ and $k_{B} \rightarrow 5$ unknowns;

$\delta_{1}$ to $\delta_{N} \rightarrow N$ unknowns;

The number of equations is equal to $3 N$, where $N$ is the total number of measured points. Therefore, the system is undetermined. Hence, it is impossible to find a solution for the system shown in Eq.(13) by means of conventional numerical methods. A more powerful and specific numerical technique is required to provide a satisfactory solution. Techniques such as orthonormal-triangular decomposition $(Q R)$ or singular value decomposition $(S V D)$ can be applied.

There are basically two ways of generating a larger number of equations. The first one would require the use of more than three sensors. The second one, which will be presented further, relies on the inclusion of experimental data from various runs in a single system. Since experimental data differ from run to run, such system is made up of linearly independent equations, which can be solved. The proposed methodology, which represents an innovative approach regarding traditional multi-probe error separation methods, is presented next. 


\section{Data Redundancy and Numerical Solution of the Model}

A mathematical model that comprises data sets from $M$ runs can be derived from the system shown in Eq.(13) simply by putting together additional equations that were generated by the new data sets:

$$
\begin{aligned}
& S_{A_{1}}^{j}=R_{P_{0}}^{j}+C A_{P}{ }^{j}+R_{R_{1}}^{j}+C A_{R}^{j}-\delta_{1}^{j} \\
& S_{A_{2}}{ }^{j}=R_{R}{ }^{j}+C A P^{j}+A P^{j}+R_{R_{2}}{ }^{j}+C A R^{j}+A_{R}{ }^{j}-\delta_{2}{ }^{j} \\
& S_{A_{3}}{ }^{j}=R_{P_{2}}{ }^{j}+C A A^{j}+2 A_{P}{ }^{j}+R_{R 3}{ }^{j}+C A_{R}{ }^{j}+2 A_{R}{ }^{j}-\delta_{3}{ }^{j} \\
& S_{A_{4}}{ }^{j}=R_{P_{3}}{ }^{j}+C A P^{j}+3 A_{P}{ }^{j}+R_{R}{ }^{j}+C A_{R}{ }^{j}+3 A_{R}{ }^{j}-\delta_{4}{ }^{j} \\
& \begin{array}{c}
\vdots \\
S_{A_{N}}{ }^{j}=R_{P_{N-1}}{ }^{j}+C A_{P}{ }^{j}+\left[A_{P}{ }^{j} \cdot(N-1)\right]+R_{R}{ }^{j}+\left[C A_{R}{ }^{j}+A_{R}{ }^{j}(N-1)\right]-\delta_{N}{ }^{j} \\
\hdashline S_{B} j^{j}=R_{P}{ }^{j}+C A P^{j}+A P^{j}+R_{R}{ }^{j}+C A_{R}{ }^{j}+k_{B}{ }^{j}
\end{array} \\
& S_{B_{2}}{ }^{j}=R_{P_{2}}{ }^{j}+C A P^{j}+2 A_{P}{ }^{j}+R_{R_{2}}{ }^{j}+C A_{R}{ }^{j}+A_{R}{ }^{j}+k_{B}{ }^{j} \\
& S_{B_{3}}{ }^{j}=R_{P_{3}}{ }^{j}+C A P^{j}+3 A_{P}{ }^{j}+R_{R_{3}}{ }^{j}+C A_{R}{ }^{j}+2 A_{R}{ }^{j}+k_{B}{ }^{j} \\
& S_{B_{4}}{ }^{j}=R_{P_{4}}{ }^{j}+C A P^{j}+4 A_{P}{ }^{j}+R_{R_{4}}{ }^{j}+C A_{R}{ }^{j}+3 A_{R}{ }^{j}+k_{B}{ }^{j}
\end{aligned}
$$

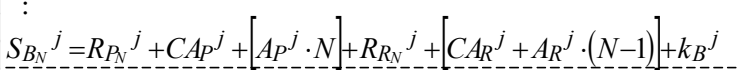

$$
\begin{aligned}
& S_{C_{1}}{ }^{j}=R_{P_{2}}{ }^{j}+C A P{ }^{j}+2 A P{ }^{j}+R_{R_{1}}{ }^{j}+C A_{R}{ }^{j}+\delta_{1}{ }^{j} \\
& S_{C_{2}}{ }^{j}=R_{P_{3}}{ }^{j}+C A P^{j}+3 A_{P}{ }^{j}+R_{R_{2}}{ }^{j}+C A_{R}{ }^{j}+A_{R}{ }^{j}+\delta_{2}{ }^{j} \\
& S_{C_{3}}{ }^{j}=R_{P_{4}}{ }^{j}+C A P^{j}+4 A_{P}{ }^{j}+R_{R_{3}}{ }^{j}+C A_{R}{ }^{j}+2 A_{R}{ }^{j}+\delta_{3}{ }^{j} \\
& S_{C_{4}}{ }^{j}=R_{P_{5}}{ }^{j}+C A_{P}{ }^{j}+5 A_{P}{ }^{j}+R_{R 4}{ }^{j}+C A_{R}{ }^{j}+3 A_{R}{ }^{j}+\delta_{4}{ }^{j} \\
& S_{C_{N}}{ }^{j}=R_{P_{N+1}}{ }^{j}+C A P^{j}+\left[A_{P}{ }^{j} \cdot(N+1)\right]+R_{R N}{ }^{j}+\left[C A_{R}{ }^{j}+A_{R}{ }^{j}(N-1)\right]+\delta_{N}{ }^{j} \\
& j=1,2, . ., M
\end{aligned}
$$

where index $j$ designates the run which a given group of equations and respective parameters belong to.

The system shown in Eq.(14) presents $M(3 N)$ equations and $M(3 N+7)$ unknowns, i.e., both the number of equations and unknowns were increased by the same proportion with relation to the system in Eq.(13). Therefore, the system in Eq.(14) can not be solved by traditional numerical methods, either. However, it is possible to reduce the number of unknowns by assuming that the test conditions remain unaltered during the whole measuring procedure, hence the straightness error of the workpiece must be the same at any run and thus the number of unknowns is reduced by $(M-1)^{*}(N+2)$. The following system is then obtained:

$$
\begin{aligned}
& \int S_{A_{1}}^{j}=R_{P_{0}}+C A_{P}{ }^{h}+R_{R_{1}}{ }^{k}+C A_{R}{ }^{s}-\delta_{1}{ }^{t} \\
& S_{A_{2}}{ }^{j}=R_{P}+C A_{P}{ }^{h}+A_{P}{ }^{h}+R_{R_{2}}{ }^{k}+C A_{R}{ }^{s}+A_{R}{ }^{s}-\delta_{2}{ }^{t} \\
& S_{A_{3}}{ }^{j}=R_{P_{2}}+C A_{P}{ }^{h}+2 A_{P}{ }^{h}+R_{R_{3}}{ }^{k}+C A_{R}{ }^{s}+2 A_{R}{ }^{s}-\delta_{3}{ }^{t} \\
& S_{A_{4}}{ }^{j}=R_{P_{3}}+C A P^{h}+3 A P^{h}+R R_{4}{ }^{k}+C A_{R}{ }^{s}+3 A_{R}{ }^{s}-\delta 4^{t}
\end{aligned}
$$

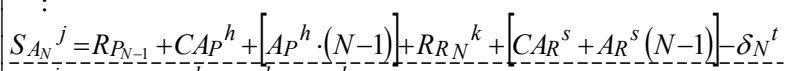

$$
\begin{aligned}
& S_{B_{1}}{ }^{j}=R_{P}+C A_{P}{ }^{h}+A P{ }^{h}+R_{R_{1}}{ }^{k}+C A_{R}{ }^{s}+k_{B}{ }^{v} \\
& S_{B_{2}}{ }^{j}=R_{P_{2}}+C A_{P}{ }^{h}+2 A_{P}{ }^{h}+R_{R_{2}}{ }^{k}+C A_{R}{ }^{s}+A_{R}{ }^{s}+k_{B}{ }^{v} \\
& S_{B_{3}}{ }^{j}=R_{P_{3}}+C A_{P}{ }^{h}+3 A_{P}{ }^{h}+R_{R_{3}}{ }^{k}+C A_{R}{ }^{s}+2 A_{R}{ }^{s}+k_{B}{ }^{v} \\
& S_{B_{4}}{ }^{j}=R_{P_{4}}+C A_{P}{ }^{h}+4 A_{P}{ }^{h}+R_{R_{4}}{ }^{k}+C A_{R}{ }^{s}+3 A_{R}{ }^{s}+k_{B}{ }^{v}
\end{aligned}
$$

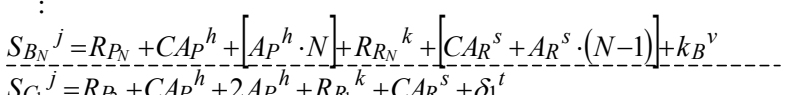

$$
\begin{aligned}
& S_{C_{2}}{ }^{j}=R_{P_{3}}+C A_{P}{ }^{h}+3 A_{P}{ }^{h}+R_{R_{2}}{ }^{k}+C A_{R}{ }^{s}+A_{R}{ }^{s}+\delta_{2}{ }^{t} \\
& S_{C_{3}}{ }^{j}=R_{P_{4}}+C A_{P}{ }^{h}+4 A_{P}{ }^{h}+R_{R 3}{ }^{k}+C A_{R}{ }^{s}+2 A_{R}{ }^{s}+\delta_{3}{ }^{t} \\
& S_{C_{4}}{ }^{j}=R_{P_{5}}+C A_{P}{ }^{h}+5 A_{P}{ }^{h}+R_{R 4}{ }^{k}+C A_{R}{ }^{s}+3 A_{R}{ }^{s}+\delta{ }^{t} \\
& S_{C_{N}}{ }^{j}=R_{P_{N+1}}+C A_{P}{ }^{h}+\left[A_{P}{ }^{h} \cdot(N+1)\right]+R_{R N}{ }^{k}+\left[C A_{R}{ }^{s}+A_{R}{ }^{s}(N-1)\right]+\delta_{N}{ }^{t} \\
& j=1,2, \ldots, M
\end{aligned}
$$

where:

$h=0$ or $h=1$ or $h=M$ is the number of reference lines for the workpiece profile;

$k=1$ or $k=M$ is the number of measuring system translational error motion sets;

$s=0$ or $s=1$ or $s=M$ is the number of reference lines for the robot error motion;

$t=0$ or $t=1$ or $t=M$ is the number of scanning device angular error sets;

$v=0$ or $v=1$ or $v=M$ is the number of zero-adjustment errors of the central sensor.

The system shown in Eq.(15) comprises an adjustable error model. The indices $h, k, s, t$ and $v$ can be appropriately chosen to set the error model to meet specific measuring conditions. Considering the determination of only one workpiece profile using data from multiple runs, the system in Eq.(15) becomes overdetermined and can be solved by means of the Least Squares fitting method.

The system shown in Eq.(15) can be rewritten in matrix form:

$$
\{S\}=[C] \cdot\{P\}
$$

where:

$\{S\}$ is a column vector composed by the readings of the three sensors, detailed below;

$$
\{S\}=\left[\left\{S_{A}\right\}^{1}\left\{S_{B}\right\}^{1}\left\{S_{C}\right\}^{1}\left\{S_{A}\right\}^{2}\left\{S_{B}\right\}^{2}\left\{S_{C}\right\}^{2} \cdots\left\{S_{A}\right\}^{M}\left\{S_{B}\right\}^{M}\left\{S_{C}\right\}^{M}\right]^{T}
$$

Each element $\left\{S_{A}\right\}^{j}$ is defined as:

$$
\left\{S_{A}\right\}^{j}=\left[S_{A 1}^{j} S_{A 2}{ }^{j} \cdots S_{A N}{ }^{j}\right]
$$

and $S_{A_{i}}{ }^{j}$ is the $i^{\text {th }}$ reading of sensor $A$ at the $j^{\text {th }}$ run.

$\{P\}$ is a column vector composed by the system parameters to be determined $\left(R_{P}, C A_{P}, A_{P}, R_{R}, C A_{R}, A_{R}, \delta_{1}, k_{B}\right)$;

$$
\{P\}=\left[\left\{R_{P}\right\}\left\{C A_{P} A_{P}\right\}\left\{R_{R}\right\}\left\{C A_{R} A_{R}\right\}\{\delta\}\left\{k_{B}\right\}\right]^{T}
$$

where:

$$
\begin{aligned}
& \left\{R_{P}\right\}=\left[R_{P_{0}} R_{P_{1}} R_{P_{2}} \cdots R_{P_{N+1}}\right] \\
& \left\{C A_{P} A_{P}\right\}=\left[C A_{P}{ }^{1} A_{P}{ }^{1} C A_{P}{ }^{2} A_{P}{ }^{2} \cdots C A_{P}{ }^{h} A_{P}{ }^{h}\right] \\
& \left\{R_{R}\right\}=\left[\left\{R_{R}\right\}^{1}\left\{R_{R}\right\}^{2} \cdots\left\{R_{R}\right\}^{k}\right] \\
& \left\{R_{R}\right\}^{k}=\left[R_{R_{1}}{ }^{k} R_{R_{2}}{ }^{k} \cdots R_{R_{N}}{ }^{k}\right] \\
& \left\{C A_{R} A_{R}\right\}=\left[C A_{R}{ }^{1} A_{R}{ }^{1} C A_{R}{ }^{2} A_{R}{ }^{2} \cdots C A_{R}{ }^{s} A_{R}{ }^{s}\right] \\
& \{\delta\}=\left[\{\delta\}\{\delta\}^{2} \cdots\{\delta\}^{t}\right] \\
& \{\delta\}^{t}=\left[\delta_{1}^{t} \delta_{2}^{t} \cdots \delta_{N}^{t}\right] \\
& \left\{k_{B}\right\}=\left[k_{B}{ }^{1} k_{B}{ }^{2} \cdots k_{B}{ }^{v}\right]
\end{aligned}
$$

and $[C]$ is the coefficients matrix of parameters $\{P\}$. The coefficients matrix is rectangular and is defined by the system depicted in Eq.(15).

After the system in Eq.(16) is obtained, a solution is needed for the sought parameters. In this case, however, a traditional solution of the model presents some drawbacks. An analysis of the system reveals that matrix $[C]$ is rectangular, ill-conditioned and rankdeficient. Thus, obtaining a satisfactory solution for the modelled 
system by means of conventional techniques is not feasible due to the above-mentioned limitations of matrix $[C]$. In this work, the orthogonal-triangular decomposition of matrices $(Q R)$ is employed to solve Eq.(16).

The $Q R$ decomposition, which is pre-programmed in numerical routines package MATLAB $\AA$, is appropriate for providing solution for rectangular sparse systems. The proposed approach differs from previous studies by its low susceptibility to environmental variations and its effectiveness on determining workpiece profile, even when the system errors are one order of magnitude greater than workpiece profile.

\section{The Measuring System and Experimental Results}

The Automated and Dedicated Measuring System (ADMsys) was developed around an ABB IRB140 six-axis articulated industrial robot. The robotic arm can handle up to $5 \mathrm{~kg}$ payload with $810 \mathrm{~mm}$ reach, which is plenty enough for the application. The manipulator control system is provided with analogue-digital (A/D) conversion circuit board for communication with peripheral equipment and signal acquisition/processing algorithms.

A probing device for straightness measurement was built to hold three specifically arranged LVDT type sensors. The distance between consecutive sensors is $18 \mathrm{~mm}$, which corresponds to the measuring step, so as to match the error model. Measuring force is equal to $0.63 \mathrm{~N} \pm 25 \%$ and uncertainty is equal to $\pm 1 \mu \mathrm{m}$.

The probing device mechanical set-up only admits a fairly reasonable adjustment of sensors positioning, in such a way that all sensors present simultaneously enough operating range to enable measurement. The previously described error model shall compensate the remaining zero-adjustment error.

All three sensors were connected to a TESA electronic measuring column for signal demodulation. The measuring column can accommodate up to four sensors and provides independent analogue output signals, which are connected to the $\mathrm{A} / \mathrm{D}$ acquisition board input channels by means of a parallel cable.

Figure 5 shows the measuring system.

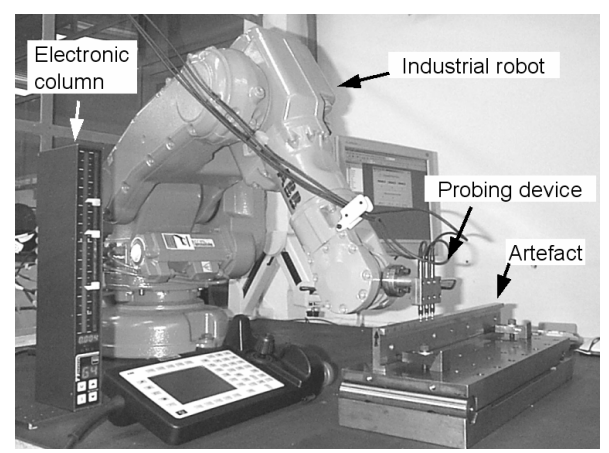

Figure 5. The Automated and Dedicated Measuring System.

The 12-bit A/D acquisition board total operating range is $600 \mu \mathrm{m}$, with resolution of approximately $0.15 \mu \mathrm{m}$ per A/D level, which is almost equal to the resolution of the sensor itself $(0.1 \mu \mathrm{m}$, as stated by the manufacturer). Since in dimensional metrology it is frequently accepted that the repeatability of an instrument corresponds to ten times its resolution, it is expected that ADMsys is able to produce satisfactory results at a limit of about $1.5 \mu \mathrm{m}$. When considered all surrounding imperfections, such as vibration from the manipulator servomotors over sensors and the table where the whole system is placed, as well as temperature variation over sensor readings, the system global uncertainty is approximately $3 \mu \mathrm{m}$.
One granite straightedge plus two steel artefacts were employed at the experimental tests. Both steel artefacts had been machined to present intentionally pronounced and known straightness errors. The measuring procedure was started with the robot activation via $\mathrm{AD} / \mathrm{DA}$ interface in order to place the probing system at the measuring path starting point on the artefact. Subsequently, the robot was moved along the artefact longitudinal direction at $18 \mathrm{~mm}$ steps and sensor readings were collected at each step. All artefacts were measured at 23 points along the measuring path and a total of five forward and five backward runs were accomplished. Further information on operational aspects of the measuring system can be found in Paziani (2005).

As stated before, the error separation model is configurable to suit a diverse number of measurement parameters. In this work, two different model configurations were employed. The first one, entitled the Complete Model, considers that all parameters of Eq.(15) vary from run to run. In other words, the complete model considers that parameters indexed by $h, k, s, t$ and $v$ of Eq.(15), i.e., are different at each run. On the other hand, in the entitled Simplified Model, the parameter given by $h$ is considered constant, i.e., the workpiece profile reference line does not vary during the whole measuring process. Next, experimental results from the application of both models are presented.

A Mitutoyo granite straightedge was measured with the purpose of testing the behaviour of the measuring system at its uncertainty limits. To begin with, the straightedge was measured by means of the reversal method, which yielded a straightness error $R_{P}$ value equal to $1.5 \pm 1.0 \mu \mathrm{m}(2 \sigma)$. Since accuracy level of the employed industrial robot lies around $100 \mu \mathrm{m}$, the straightedge can be taken as a reference for the system.

Next, the granite straightedge was measured by the proposed system. The solution using the complete model yielded a straightness error of $3.9 \pm 8.8 \mu \mathrm{m}(2 \sigma)$. The application of the simplified model to the measurement of the granite straightedge produced a straightness error equal to $3.6 \pm 8.6 \mu \mathrm{m}(2 \sigma)$. The difference between measurements by ADMsys and reversal can be ascribed to the fact that the measured quantity leans too close to the uncertainty of the proposed system, which is approximately $3 \mu \mathrm{m}$. In such situations, the measuring system is prone to error sources that were not considered in the models, as vibration, for instance. Figures 6 and 7 show the measurements of the granite straightedge using the ADMsys complete model and simplified model, respectively, compared to the reversal method.

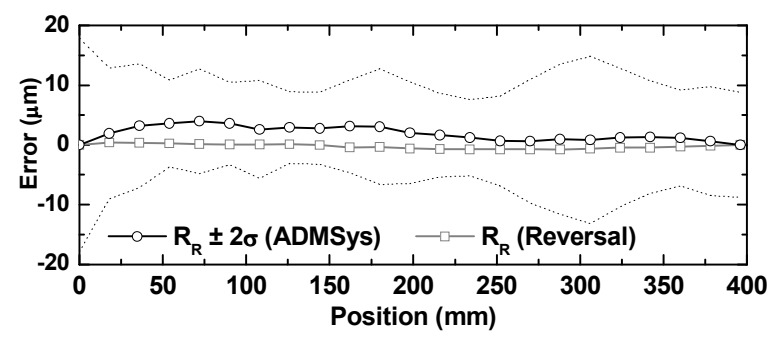

Figure 6. Straightness error of granite straightedge - Complete Model.

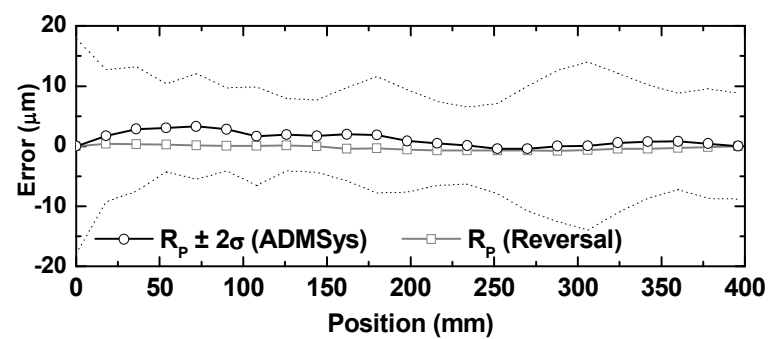

Figure 7. Straightness error of granite straightedge - Simplified Model. 
Figure 8 below shows the artefact No 1 straightness error, which was obtained by means of the complete model, compared to the reversal method:

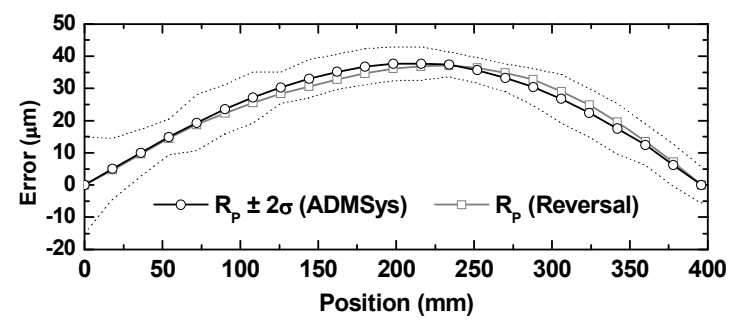

Figure 8. Straightness error of artefact No 1 - Complete Model.

The measurement of artefact $\mathrm{N}^{\mathrm{o}} 1$ straightness error $R_{P}$ by the complete model yielded $37.7 \pm 6.6 \mu \mathrm{m}(2 \sigma)$. In contrast, the reversal method yielded $37.1 \pm 2.0 \mu \mathrm{m}(2 \sigma)$. For aesthetics reasons, standard deviation for the reversal method is not shown in the graph of Fig. 8. The small difference between the maximum values of the presented errors is equal to $0.6 \mu \mathrm{m}$, which corresponds to a percent error of $1.59 \%$, and indicates the satisfactory character of the decoupling process. Average errors are equal to $23.2 \mu \mathrm{m}$ (ADMsys) and $23.0 \mu \mathrm{m}$ (reversal) and emphasize the effectiveness of the proposed method. The overall decoupling result of $R_{P}$ can be considered good.

The straightness error of artefact $\mathrm{N}^{\mathrm{o}} 1$ by means of the simplified model is presented in Fig. 9, below:

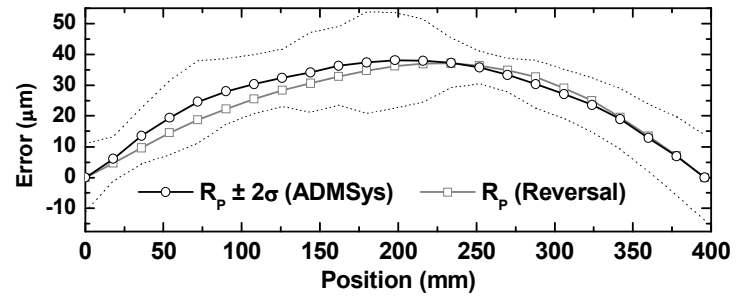

Figure 9. Straightness error of artefact No 1 - Simplified Model.

Artefact № 1 straightness error $R_{P}$, using ADMsys simplified model, is equal to $38.1 \pm 10.5 \mu \mathrm{m}(2 \sigma)$, whilst the reversal method yielded $37.1 \pm 2.0 \mu \mathrm{m}(2 \sigma)$. The difference between maximum errors is equal to $1 \mu \mathrm{m}$, which corresponds to a percent error of $2.6 \%$ and indicates that the separation process is satisfactory. The maximum difference between profiles $(5.8 \mu \mathrm{m})$ is larger than the obtained with the complete model $(2.5 \mu \mathrm{m})$, but the small difference between average errors, nearly $1.5 \mu \mathrm{m}$, suggests good repeatability. Average straightness errors are $24.5 \mu \mathrm{m}$ and $23.0 \mu \mathrm{m}$ for ADMsys and reversal method, respectively.

Figure 10 below shows the measurement results of artefact $\mathrm{N}^{\mathrm{o}} 2$. The profile straightness error $R_{P}$ by means of the complete model is equal to $111.4 \pm 1.5 \mu \mathrm{m}(2 \sigma)$, whereas the reversal method yielded $115.9 \pm 4.0 \mu \mathrm{m}(2 \sigma)$. The difference between maximum errors is $4.5 \mu \mathrm{m}$, which is equivalent to a percent error of $3.9 \%$.

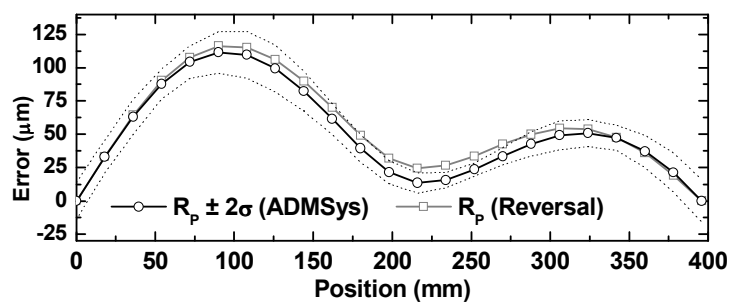

Figure 10. Straightness error of artefact No 2 - Complete Model.
In this case, good correspondence between profile shapes can be observed. The improved shape matching may be credited to the fact that artefact № 2 straightness error magnitude is about the same as robot translational error motion (approximately $140 \mu \mathrm{m}$, not shown), allowing better numerical solution of the model.

The results for artefact $\mathrm{N}^{\mathrm{o}} 2$ error decoupling are presented next.

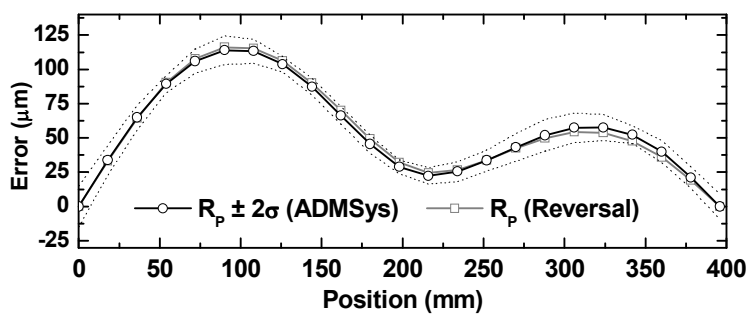

Figure 11. Straightness error of artefact No 2 - Simplified Model.

As a result of the application of the simplified measuring model, profile straightness error of artefact № $2, R_{P}$, is equal to $113.9 \pm$ $8.2 \mu \mathrm{m}(2 \sigma)$, while the reversal method yielded $115.9 \pm 4.0 \mu \mathrm{m}$ $(2 \sigma)$. Percent error between curves of Fig. 11 is equal to $1.7 \%$, which corresponds to less than half the percent error obtained for artefact $\mathrm{N}^{\mathrm{o}} 2$ using the complete model and reveals the adequacy of the adjustable error model to suit different measuring conditions.

Average errors are $54.7 \mu \mathrm{m}$ and $54.9 \mu \mathrm{m}$, obtained by the proposed system and reversal method, respectively, which demonstrate high-quality decoupling ability.

\section{Conclusions}

In this work, an Automated and Dedicated Measuring system (ADMsys) was developed aiming at the measurement of straightness errors of mechanical parts.

An industrial robot that operates specifically arranged displacement sensors comprises the proposed system. An error separation technique was employed to decouple part error from errors induced by the measuring system itself. An innovative error model, which takes into consideration the zero-adjustment errors of sensors, was developed and provided profile identification in one operation only.

Since the error model demands three equally spaced sensors, a probing device was built accordingly. Three LVDT type sensors were employed and provided adequate resolution to meet most common machining processes precision requirements.

An electronic interface was implemented to acquire displacement data and to perform a communication path between the robot control system and a microcomputer in order to activate programmed robot movements. The interface comprises an AD/DA acquisition board, object-oriented software and electric connections.

The error separation algorithm was developed using MATLAB programming environment. The solution of an ill-conditioned linear system of equations was accomplished by means of the application of $Q R$ decomposition, which allowed the solution of the sparse least squares problem.

Experimental tests were performed to verify the efficiency of the error separation algorithms. The tests consisted of measuring granite straightedge, as well as two steel artefacts presenting intentionally machined straightness errors. The results obtained from the proposed system were compared to the ones provided by the reversal method upon the same artefacts.

The following essential points can be highlighted:

- The proposed system is mechanical and electronically stable and can possibly be employed at industrial environments; 
- Experimental tests with the proposed system were completed much faster than with the application of the reversal method. The automated straightness measurement took about 12 minutes, whilst the measurement by the reversal technique took nearly 30 minutes;

- ADMsys set-up cost is $75 \%$ the value of a small size CNC coordinate measuring machine. In industries with previously installed robots, cost is reduced by $90 \%$, if the same model used in this work is considered.

- ADMsys eliminates the influence of the human operator upon results and besides, allows full-lot inspection aiming selective assembly;

- An adjustable error model was developed so as to suit different measurement conditions;

- The reference coordinate system proposed to the error model allowed artefact straightness error decoupling so that the deleterious influence of the zero-adjustment error of sensor B was minimized;

- The solution of a matrix error model by means of QR decomposition provided the results of all variables in one operation only and does not make use of substitution in other equations of the system, nor performs numerical integration and/or differentiation. Therefore, the solution is free from both cumulative errors along the measuring path and large numerical processing errors.

\section{Acknowledgements}

The authors would like to express their gratitude to Fundação de Amparo à Pesquisa do Estado de São Paulo - FAPESP, and Conselho Nacional de Desenvolvimento Científico e Tecnológico CNPq, who have supported this project.

\section{References}

Boillot, J.P. \& Uota, K., 2002, "Flexible robotic measuring of weldments on production lines", Industrial Robot: An International Journal, Vol. 29, No. 1, pp. 43-48.

Bryan, J.B. \& Carter, D.L., 1989, "How straight is 'straight?", American Machinist, No. 133, pp. 61-65.

Evans, C.J., Hocken, R.J. and Estler, W.T., 1996, "Self-calibration: reversal, redundancy, error separation, and 'absolute testing", Annals of the CIRP, Vol. 45, No. 2, pp. 617-634.
Gao, W. \& Kiyono, S., 1996, "High accuracy profile measurement of a machined surface by the combined method", Measurement, Vol. 19, No. 1, pp. 55-64.

Gao, W. \& Kiyono, S., 1997, "On-machine profile measurement of machined surface using the combined three-point method", JSME International Journal, Series C, Vol. 40, No. 2, pp. 253-259.

Gao, W., Yokoyama, J., Kojima, H. and Kiyono, S., 2002, "Precision measurement of cylinder straightness using a scanning multi-probe system", Precision Engineering, Vol. 26, No. 3, pp. 279-288.

Grant, M.B., 1995, "Financial evaluations" In: Bosch, J.A. (Ed.), "Coordinate measuring machines and systems", Marcel Dekker, New York, Cap. 14, pp. 403-426.

Greenway, B., 2000, "Robot accuracy", Industrial Robot: An international Journal, Vol. 27, No. 4, pp. 257-265.

Hocken, R.J., 1995, "Measurement integration”, In: Bosch, J.A. (Ed.), "Coordinate measuring machines and systems", Marcel Dekker, New York, Cap.13, pp.391-402.

Hoshizaki, J. \& Bopp, E., 1990, "Robot applications design manual", John Wiley \& Sons, New York.

Kato, H., Sone, R.Y. and Nomura, Y., 1991, "In-situ measuring system of circularity using an industrial robot and a piezoactuator", International Journal of the Japan Society of Precision Engineering, Vol. 25, No. 2, pp.130-135.

Lee, K.H. \& Park, H.P., 2000, “Automated inspection planning of freeform shape parts by laser scanning", Robotics and Computer Integrated Manufacturing, Vol. 16, No. 4, pp. 201-210.

Mitsui, K., 1982, "Development of a new measuring method for spindle rotation accuracy by the three points method", Proceedings of the $23^{\text {rd }}$ International MTDR Conference, pp.115-121.

Paziani, F. T., 2005, "Development of an automated and dedicated measuring system" (In Portuguese), Ph.D. Thesis, Escola de Engenharia de São Carlos, Universidade de São Paulo, São Carlos, SP, Brazil.

Picard, M.P., 2002, "Flexplace: watchmaker precision for robotic placement of automobile body parts", Industrial Robot: An International Journal, Vol. 29, No. 4, pp. 329-333.

Tanaka, H., Tozawa, K., Sato, H., O-hori, M. and Sekiguchi, H., 1981, "Application of a new straightness measurement method to large machine tool", Annals of the CIRP, Vol. 30, No. 1, pp. 455-459.

Tanaka, H. \& Sato, H., 1986, "Extensive analysis and development of straightness measurement by sequential-two-points method", Journal of Engineering for Industry, Vol. 108, No. 3, pp. 176-182.

Whitehouse, D.J., 1976, "Some theoretical aspects of error separation techniques in surface metrology", Journal of Physics E: Scientific Instruments, Vol. 9, No. 7, pp. 531-536.

Young, K. \& Pickin, C.G., 2000, "Accuracy assessment of the modern industrial robot", Industrial Robot: An international Journal, Vol. 27, No. 6 , pp. 427-436. 